\title{
Eradicating Social Isolation: A Grand Challenge for Social Work
}

\author{
Suzanne Brown ${ }^{1}$
}

Published online: 4 February 2020

(c) Springer Science+Business Media, LLC, part of Springer Nature 2020

\section{Position and Fact Sheet}

Social isolation is a silent killer-as dangerous to health as smoking. National and global health organizations have underscored the hidden, deadly, and pervasive hazards stemming from feeling alone and abandoned. Our challenge is to educate the public on this health hazard, encourage health and human service professionals to address social isolation, and promote effective ways to deepen social connections and community for people of all ages.

\section{The Issue}

Americans are more socially isolated now than ever before. Research finds that it can be a silent yet potent killer that affects diverse groups of Americans across the age spectrum. The high correlation between social isolation and health issues, including morbidity and mortality has led this hazard to become one of the priorities for social work research and intervention.

\section{Societal Goal}

Through interdisciplinary collaboration and innovative interventions, we can reduce the risk of social isolation across all populations and strengthen social ties that are a vital source of emotional strength and health improvement. The "social" element of social work is the key for reducing the risk of social isolation and strengthening social ties among all populations.

\section{A Decade of Work}

- Increase access to high quality childcare that strengthens social connectedness for both children and caregivers.

- Build more age-friendly communities that strengthen frayed social ties and prevent elderly mistreatment.

- Advocate for reform of solitary confinement.

- Implement assessment protocols in healthcare settings that encompass social health status as an attribute presented when seeking care and treatment.

- Increase research on social isolation among marginalized communities using a multisystems approach at individual, familial, community, and societal levels.

- Address the isolating consequences of hearing loss and other sensory impairments.

- Create opportunities for social ties in large portions of the population through expanding evidence-based interventions that harness the power and access of technology.

Publisher's Note Springer Nature remains neutral with regard to jurisdictional claims in published maps and institutional affiliations.
2018 Grand Challenge Fact Sheet No. 5: Eradicate Social Isolation. (January, 2018). Retrieved From https://grandchallenges forsocialwork.org/eradicate-social-isolation/.

Suzanne Brown

suzanne.brown@wayne.edu

1 Wayne State University School of Social Work, 5447 Woodward Avenue, Detroit, MI 48202, USA 